\title{
Stealth Ships Detection by using Laplacian of Gaussion Algorithm
}

\author{
Sagar Shankarrao Dake \\ M.Tech (IT), Gitam University \\ Visakhapatnam, \\ India
}

\begin{abstract}
:
In last few years, the stealth technology are designed to avoid detection using a variety of advanced technologies that reduce reflection/emission of radar, infrared, visible light, radio-frequency (RF) spectrum, and audio, collectively known as stealth technology. In this paper, the stealth technology ships will be easily detected by Laplacian of Gaussian algorithm.
\end{abstract}

\section{Keywords}

DCT, DHT, Sobel operator, 3D edge detector.

\section{INTRODUCTION:}

Stealth technology also termed LO technology (low observable technology) is a sub-discipline of military tactics and passive electronic countermeasures, which cover a range of techniques used with personnel, aircraft, ships, submarines, missiles and satellites to make them less visible (ideally invisible) to radar, infrared, sonar and other detection methods. Stealth is one of the most misunderstood and misinterpreted concepts in military aviation by the common man. Stealth aircraft are considered as invisible aircraft, which dominate the skies. With an additional boost from Hollywood action movies, stealth is today termed as the concept invincibility rather than invisibility. Though, the debate still continues on whether stealth technology can make an aircraft invincible it was found that stealth aircraft are detectable by radar. The motive behind incorporating stealth technology in an aircraft is not just to avoid missiles being fired at is but also to give total deniability to covert operations. This is very much useful to strike targets where it is impossible to reach. In this paper, the stealth ships will be easily detected by using Laplacian algorithm. The general design of a stealth aircraft is always aimed at reducing radar and thermal detection. It is the designer's top priority to satisfy the following conditions; some of which are listed below, by using their skills, which ultimately decides the success of the aircraft:-

- $\quad$ Reducing thermal emission from thrust

- Reducing radar detection by altering some general configuration

- Reducing radar detection when the aircraft opens its weapons bay

- $\quad$ Reducing infra-red and radar detection during adverse weather conditions

One of the possible definition is as follow: stealth technology minimize the observable aspect of a piece of military equipment, including radar and infrared signature, visibility and sound. Stealth technology is used to make military equipment more difficult to detect, track, identify and engage by defensive weapon system. Signature control or stealth is nothing new to aircraft, armies and navies.
Stealth Technology in Ship:

Continuous developing in military ships technology by stealth technology have produce a new sort of defensive weapon. Stealth ships can easily invisible into enemy, drop a payload and come back out without being detected, identified or attacked.

To get these goals, the stealth in many ways: [1]

1) It must be very hard to detect on radar

2) Its engine should not produce heat or smoke

3) It must be quite.

4) It should be very hard to see with human eye.

\section{RELATED WORK}

\subsection{Image Noise Reduction:}

Median Filter [2]

The median filter is normally used to reduce noise in an image and it is a simple and very effective noise removal filtering process. Noise is removed by replaces the center value with the median of all the pixel values in the matrix. If we considering an example of $3 \times 3$ matrix.

Table 1

\begin{tabular}{|l|l|l|}
\hline 11 & 15 & 19 \\
\hline 12 & 17 & 18 \\
\hline 14 & 16 & 13 \\
\hline
\end{tabular}

Table 2

\begin{tabular}{|l|l|l|}
\hline$*$ & $*$ & $*$ \\
\hline$*$ & 15 & $*$ \\
\hline$*$ & $*$ & $*$ \\
\hline
\end{tabular}

The median filter sorts the value of the given matrix and then median value is replace by center value. The sorted values are $11,12,13,14,15,16,17,18,19$ and median value 15 will be replaced by centre value in the matrix. This process is performed over the whole image and reduces the noise.

\subsection{JPEG compression methods:}

The image compression it refer to minimize the bits require to represent an image. Images are used for transmission and storage of information. The main role of image compression is that to compress the image which is used in broadcast television, remote sensing, military 
communication and facsimile transmission. Storage of images is needed for education and business document, medical image in computer tomography, magnetic resonance imaging, motion picture, satellite image and geological survey. The image contains a high degree of redundancy that's why compression of image data without degradation of image quality is possible. These are as: [6]

1) Special redundancy due to correlation between neighbouring pixels.

2) Spectral redundancy due to correlation between colour components.

3) Psychovisual redundancy due to properties of human visual system.

An image compression system consists of three basic blocks such as transformer, quantizer and coder. The transformer takes the raw image data and provide image. The quantizer generates limited number of symbol that is representation of compress image. The coder assigns a code word to each symbol, which may be of fixed length. JPEG scheme is essentially employ the discrete cosine transformer (DCT) representation of the image and representation scheme using a real value discrete Harley transform (DHT).

\subsubsection{JPEG Compression Using DCT [3]}

The image is first partitioned into different blocks. Discrete Cosine Transform (DCT) is applied to each of the blocks to convert from spatial domain to frequency domain. The transform coefficients using the DHT of a block of pixels $\mathrm{x}(\mathrm{m}, \mathrm{n})$ may be obtained as

$$
\begin{aligned}
& D(i, j) \\
& =\frac{1}{\sqrt{2 n}} c(i) c(j) \sum_{x=0}^{N-1} \sum_{y=0}^{N-1} p(x, y) \cos \frac{(2 x+1) i \Pi}{2 N} \cos \frac{(2 y+1) j \Pi}{2 N}
\end{aligned}
$$

Where $p(x, y)$ is the $x$, yth element of the image represented by the matrix $\mathrm{p}$. $\mathrm{N}$ is the size of the matrix on which the DCT is applied. Hence small set of coefficients are obtained. The DCT coefficients are quantized using a quantization matrix provided by JPEG standard. The JPEG quantizer consists of 64 uniform quantizer.

The $i^{\prime}$ th quantizer is calculated as

\section{$\mathrm{Yi}=\mathrm{Round}(\mathrm{Xi} / \mathrm{Qi})$}

where Qi is the $\mathrm{i}^{\prime}$ th quantization step size, $\mathrm{Xi}$ is the input and $\mathrm{Yi}$ is the quantized version of $\mathrm{Xi}$. After quantization the quantized coefficients are arranged in a zigzag order that is arranged in lowest to highest spatial frequency. Then a lossless coding technique that is, Run-length coding is applied to further compress the data. The decoding is just the inverse of encoding process.

\subsubsection{JPEG Compression Using DHT [3]}

The forward and inverse transforms are same in DHT along with an inclusion of a scale factor in its inverse transform. Besides, the DHT can compute both convolution and the DFT efficiently. The memory requirement to compute both the forward and inverse DHT is about half as those of the DCT. The transform coefficients using the DHT of a block of /pixels $x(m, n)$ may be obtained as

$$
\begin{gathered}
X(k, l)=\sum_{n=0}^{N-1} \sum_{m=0}^{M-1} x(m, n)\left[\cos \left(2 \Pi\left(\frac{k m}{M}+\frac{l n}{N}\right)\right)\right. \\
\left.+\sin \left(2 \Pi\left(\frac{k m}{M}+\frac{l n}{N}\right)\right)\right]
\end{gathered}
$$

Where $\mathrm{k}=0,1, \ldots \mathrm{M}-1$ and $1=0,1, \ldots \mathrm{N}-1, \mathrm{x}(\mathrm{m}, \mathrm{n})$ represents the block of pixels on which the DHT is calculated. The Inverse Discrete Hartley Transform may be obtained as

$$
\begin{aligned}
X(k, l)=(1 / M N) & \sum_{n=0}^{N-1} \sum_{m=0}^{M-1} x(m, n)\left[\cos \left(2 \Pi\left(\frac{k m}{M}+\frac{l n}{N}\right)\right)\right. \\
& \left.+\sin \left(2 \Pi\left(\frac{k m}{M}+\frac{l n}{N}\right)\right)\right]
\end{aligned}
$$

Where $\mathrm{k}=0,1, \ldots . \mathrm{M}-1$ and $1=0,1, \ldots \mathrm{N}-1$. DHT coefficients do not follow zigzag scanning; they follow a special scanning. So the designing of the Quantization matrix is quite difficult in DHT. To eliminate these difficulties, energy quantization is used.

\subsection{Sobel Operator:}

The Sobel operator, sometimes called Sobel Filter, is used in image processing and computer vision, particularly within edge detection algorithms, and creates an image which emphasizes edges and transitions. In this paper the sobel operator is used for detection of ships names. The Sobel operator is based on convolving the image with a small, separable, and integer valued filter in horizontal and vertical direction and is therefore relatively inexpensive in terms of computations. The operator uses two $3 \times 3$ kernels which are convolved with the original image to calculate approximations of the derivatives - one for horizontal changes, and one for vertical. If we define $\mathbf{A}$ as the source image, and $\mathbf{G}_{x}$ and $\mathbf{G}_{y}$ are two images which at each point contain the horizontal and vertical derivative approximations, the computations are as follows

$$
\Delta f=\operatorname{mag}(\Delta f)=\left[G_{x}^{2}+G_{y}^{2}\right]^{\wedge} 1 / 2
$$

\subsection{Motion Vector Distribution [5]}

The H.264 motion vector prediction tool is define the new position as search center instead of zero motion vector. In the JVT software the predicted motion vection (PMV) is determine the search center and it choose as the initial search point from the nearest integer motion vection from PMV. The motion vector prediction uses the neighboring block motion information as a reference. In figure 1 shows the PMV of current block F is set to the median value of its adjacent block include the left $(\mathrm{G})$, the top $(\mathrm{H})$, and the top left (I) (or the top right (J) when I is not available Pred_mv = median $\left(\mathrm{mv} \_\mathrm{G}, \mathrm{mv} \_\mathrm{H}, \mathrm{mv} \_\mathrm{J}\right)$

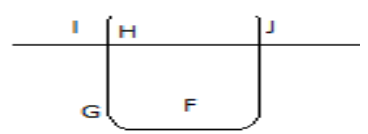

\section{Figure 1. Locations of block of reference MV and current block}

In H.264 the motion vector is quarter accuracy, many motion estimated algorithm including joint medal (JM) of H.264 and some speed-up algorithm still select the nearest integer position from pred_mv as a search center and perform the search process from integer position. This may lead to compare unnecessary search points. In fact, the predicted motion vectors are very near to the real motion vectors 


\subsubsection{Motion region identification [5]}

The first step of algorithm is motion region identification. it detect the motion region using the VRA with a little additional computation cost. The whole frame is carried out from the original VRA and the detected motion region will cross the MB border, for that region it improves VRA to adapt the result of video coding. The new VRA it work like, first it will divide the picture in to $2 \times 2$ sub picture and then VRA works on the four sub-picture respectively to acquire more efficient identification due to only one rectangle region can be mark as motion region in the original VRA, and at the final stage the four detected motion region is merged and it aligned to MB boundary. In which the alignment criterion is the number of motion pixels in MB. The more efficient ME can be performed on them since there is no or low motion in the still MBs

\section{PROCESS OF WORK:}

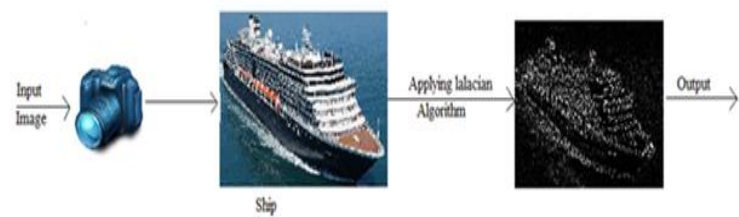

Figure 2. Process of laplacian algorithm

\section{A 3D EDGE DETECTION SCHEME} [4]

\subsection{Filtering Stage}

Choice of 1D smoothing operator: $T(X)$

We strongly recommend to choice a filter that can be implemented recursively, mainly because of the computing time. We can for example choose one of two filters: $T_{1}$ or $T_{2}$

$$
\begin{aligned}
& T_{1}(\mathrm{x})=\mathrm{ce}^{-\alpha|\mathrm{x}|} \\
& T_{2}(\mathrm{x})=(\mathrm{c}|\mathrm{x}|+1) \mathrm{e}^{-\alpha|\mathrm{x}|}
\end{aligned}
$$

Theoretically the derivation filter $T_{2}^{\prime}(\mathrm{x})$ is better than Canny's multiple response criterion, but $T l(x)$ meets the best trade-off detection location. For small value $T_{2}^{\prime}(\mathrm{x})$ induce some delocalization problem. This drawback will be share to any filter whose impulse response is at point 0 .
Or else the results do not different on many kinds of image.

\subsection{Choice of the Kind of Approach}

Generally the second derivative of the computation is more sensible to noise. At second side the computational cost is lower, because of the simplification will occurs when the computing impulse will filter by addition of smoothing and multiplication and second derivative operator. the noisy images are useful to threshold zero crossing provided by filtering stage, and at each zero crossing stage it require to compute the gradient magnitude. The location of edges provided by two kind of method is experimentally the same. The laplacian approach it may be point out to the smooth right angle.

Let $\mathrm{I}(\mathrm{X} 1, \ldots \ldots . \mathrm{Xn})$ image dimension $\mathrm{n}$.

Let $\mathrm{G}(\mathrm{X} 1, \ldots \ldots . \mathrm{Xn})$ Gradient of I.

$$
G(I)=\left(\frac{\partial I}{\partial X 1}, \ldots \ldots \frac{\partial I}{\partial X n}\right)^{t}
$$

The computation of the gradient component $\frac{\partial I}{\partial X 1}$ is done by computing image $\left(D_{i}\right)$ corresponding to the partial derivatives with respect to $X_{i}$ as follow

$$
\begin{aligned}
& \text { For } \mathrm{i}=1, \ldots \mathrm{n} \text { do } \\
& \begin{array}{l}
D_{i}=\mathrm{I} \\
\text { For j e }[1, \ldots . \mathrm{n}] \backslash\{\mathrm{i}\} \text { do } \\
D_{i}=D_{i} * \mathrm{~T}\left(X_{j}\right) \\
D_{i}=D_{i} * T^{\prime}\left(X_{1}\right)
\end{array}
\end{aligned}
$$

For an image of size $\mathrm{p}$ (ie $d_{1} * \ldots * d_{p}$ ) the computation of each gradient component needs to compute $p$ convolutions per point. The we obtain for the entire computation $p^{2} . \Pi_{x=1}^{p} d_{i}$. convolutions. If we use a direct implementation of a convolution mask ID of size $\mathrm{k}$, we obtain the following complexity: $k p^{2} \Pi_{x=1}^{p} d_{i}$. A recursive filtering of order $r$ allows to obtain a complexity of order: $r p^{2} \Pi_{x=1}^{p} d_{i}$. The computation of the second derivative may be done with the algorithmic structure precedently described where the first derivative operator $T^{\prime}(x)$ is replaced by the second derivative operator $T^{\prime \prime}(x)$. However for some cases calculus simplification occur allowing to reduce the computational cost

\section{PROPOSED WORK:}

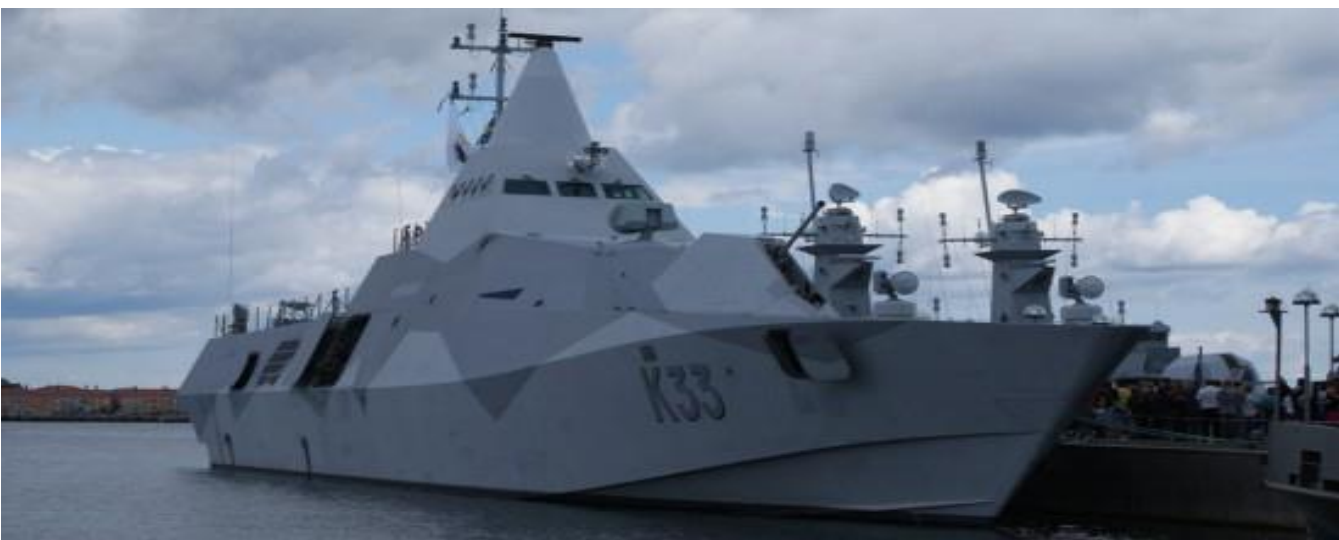

Figure 3. Original image of stealth technology 


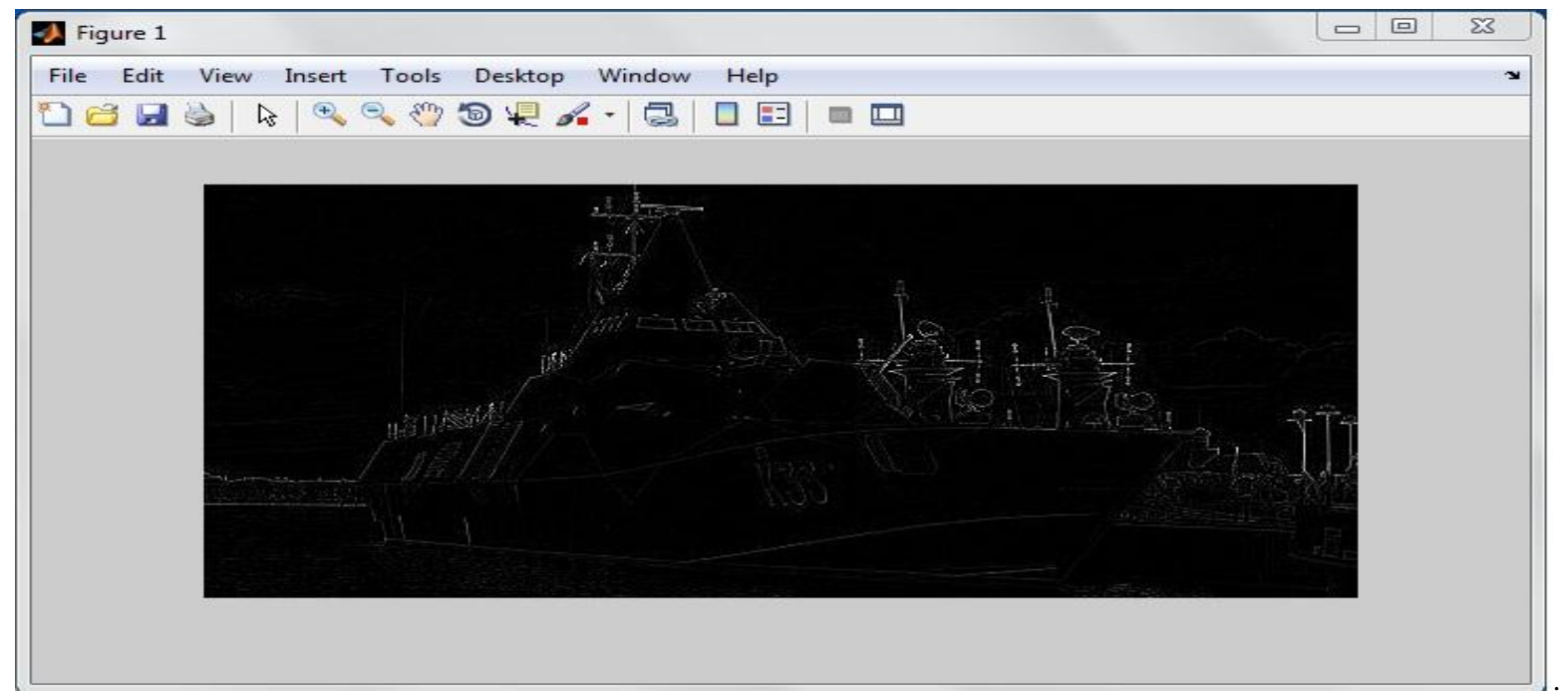

Figure 4. Output of original image.

Step 1:

In this paper, the stealth technology ship will detected by using Laplacian of Gaussian algorithm. The first stage is that the camera will take a photo $(2 \mathrm{D}$ or $3 \mathrm{D})$ then if in that image if it is noise image then image noise reduction process will start. The JPEG compression process is used to compress the image size by using DCT and DHT compression method.

Step 2:

The Sobel operator is mainly used for detection of ship name. The Sobel operator are mainly based on convolving the image with a small, separable and integer value filter in horizontal and vertical direction. In this paper, camera will take a $2 \mathrm{D}$ or $3 \mathrm{D}$ photo. When camera will take $3 \mathrm{D}$ photo then it will filter the 1D smoothing operators.

Step 3:

At final stage the stealth technology ship will detected by using Laplacian algorithm (edge detection) and the Sobel operator will detect the name of ship.

\section{ACKNOWLEDGMENTS}

The author would like to thank Dr. Ravi Bramaramba from Gitam University for various help.

\section{CONCLUSION:}

The proposal work is described for stealth ships detection by using Laplacian of Gaussian Algorithm. The digital camera images are compressed by using DHT, DCT method, sobel operator for detection of ships name and motion detector are used in this paper.

\section{REFERENCES}

[1] Zrínyi Miklós National Defence University, Electronic Warfare Department, Budapest, Hungary "Stealth technology deployed on the battlefield".
[2] Pravesh Kumar Goel, Paresh P. Kotak, Amit Gupt "Method of Moving Region Detection for Static Camera".

[3] Dhananjay Patel, Alina Menoth Jose, Nigel Mascarenhas "JPEG Image Compression using DCT and DHT and Comparison of Both Techniques based on Mean Square Error and Peak Signal to Noise Ratio".

[4] Olivier MONGA, Rachid DERICHE, Gregoire MALANDAIN, Jean Pierre COCQUEREZ "3D edge detection by separable recursive filtering and edge closing".

[5] Dongming Zhang, Advanced Computing Research Lab., Beijing Key Laboratory of Mobile, Computing and Pervasive Device, Institute of Computing Technology, Beijing,China, Gang Cao, National Application Software, Testing Labs, Beijing,China, Xiaoguang Gu, Advanced Computing Research Lab, Beijing Key Laboratory of Mobile, Computing and Pervasive Device, Institute of Computing Technology, Chinese Academy of Science, Beijing,China, "Improved Motion Estimation Based on Motion Region Identification" 2012 International Conference on Systems and Informatics (ICSAI 2012).

[6] Sunil Kumar Pattanaik' and K. K. Mahapatra Department of Electronics and Communication Engineering, NIT Rourkela, India "DHT Based JPEG Image Compression Using a Novel Energy Quantization Method". 